\title{
Musculoskeletal manifestation in the joints of the diabetic animal model Otsuka Long-Evans Tokushima Fatty rat: A histological study
}

\author{
KYOUNG SOO KIM ${ }^{1}$, IN-KYUNG JEONG ${ }^{2}$, SANG-HOON LEE ${ }^{3}$, \\ RAN SONG ${ }^{3}$, HYUNG-IN YANG ${ }^{3}$ and JUNG YEON KIM ${ }^{4}$ \\ ${ }^{1}$ East-West Bone and Joint Research Institute; ${ }^{2}$ Division of Endocrinology, Department of Internal Medicine; \\ ${ }^{3}$ Division of Rheumatology, Department of Internal Medicine, Kyung Hee University Hospital at Gangdong, \\ Gangdong-gu, Seoul; ${ }^{4}$ Department of Pathology, Inje University Sanggye Paik Hospital, \\ Nowon-gu, Seoul, Republic of Korea
}

Received October 13, 2011; Accepted December 15, 2011

DOI: $10.3892 / \mathrm{mmr} .2011 .724$

\begin{abstract}
Diabetes mellitus (DM) is a chronic metabolic disease and is associated with vascular complications. However, the association of musculoskeletal manifestations and DM is not clear. We investigated musculoskeletal manifestations in the diabetic animal model Otsuka Long-Evans Tokushima Fatty (OLETF) rat. OLETF rats and control LETO (LongEvans Tokushima Otsuka) rats at two different ages (44 and 95 weeks) were used. Knee joints and ankles with interphalangeal joints were removed, dissected, stained with hematoxylin and eosin (H\&E), periodic acid-Schiff, methenamine silver, and Masson-trichrome staining and examined under light microscopy. Mild degenerative changes with focal edema and mild fibrosis were noted in OLETF rats (at 95 weeks of age) and in age-matched LETO rats, particularly in the interphalangeal joints. Necrosis, phagocytosis of necrotic fibers, regeneration, mononuclear inflammatory cell infiltration, granulation tissue, calcification and cartilage erosion were not observed in either the aged diabetic or the non-diabetic group. We found no prominent musculoskeletal manifestations in the OLETF rats. The reasons may be due to the low prevalence rate of these anomalies, or the life span of rats may be too short to express these alterations. More studies are needed to elucidate
\end{abstract}

Correspondence to: Dr Jung Yeon Kim, Department of Pathology, Inje University Sanggye Paik Hospital, 761-1 Sanggye 7-dong, Nowon-gu, Seoul, Republic of Korea

E-mail: pck2973@yahoo.co.kr

Dr Kyoung Soo Kim, East-West Bone and Joint Research Institute, Kyung Hee University, 149 Sangil-dong, Gangdong-gu, Seoul, Republic of Korea

E-mail: labrea46@yahoo.co.kr

Key words: diabetes mellitus, Otsuka Long-Evans Tokushima Fatty rats, Long-Evans Tokushima Otsuka rats, musculoskeletal, diabetic complication the pathophysiological mechanisms by demonstrating the musculoskeletal manifestations histologically.

\section{Introduction}

Diabetes mellitus (DM) is a group of chronic metabolic diseases. DM without proper treatment results in various complications. Acute complications include hyperglycemia, diabetic ketoacidosis, or nonketotic hyperosmolar coma. Serious long-term complications include cardiovascular disease, chronic renal failure and retinal damage. Microvascular and macrovascular events are some of the most serious and extensively studied complications.

In contrast, musculoskeletal manifestations of DM are relatively rare and few reports have appeared in the literature $(1,2)$. Diabetic muscle infarction, limited joint motility, neuropathic osteoarthropathy, shoulder adhesive capsulitis, Dupuytrens disease, carpal tunnel syndrome, and stenosing flexor tenosynovitis are known to be associated with DM. Particularly, abnormalities of the hand and shoulder appear to be common among other musculoskeletal system disorders (1). Gamstedt et al reported that the prevalence of hand abnormalities increases with the duration of DM (3). Since these studies to clarify the precise pathogenic mechanisms of musculoskeletal disorders were mostly based on epidemiologic data, they are subject to limitations (4).

Otsuka Long-Evans Tokushima Fatty (OLETF) rats serve as an animal model of human type II DM, and they exhibit late-onset hyperglycemia (after 18 weeks of age), a chronic disease course, and obesity $(5,6)$. In this study we investigated musculoskeletal manifestations in OLETF diabetic rats as an explanation for the possible pathogenic mechanisms that cause hand and shoulder disorders in patients with DM.

\section{Materials and methods}

Animals. Two different age groups of male OLETF rats (seven 44-week-old rats, seven 95-week-old rats) were used in this study. As a control group, LETO rats were 

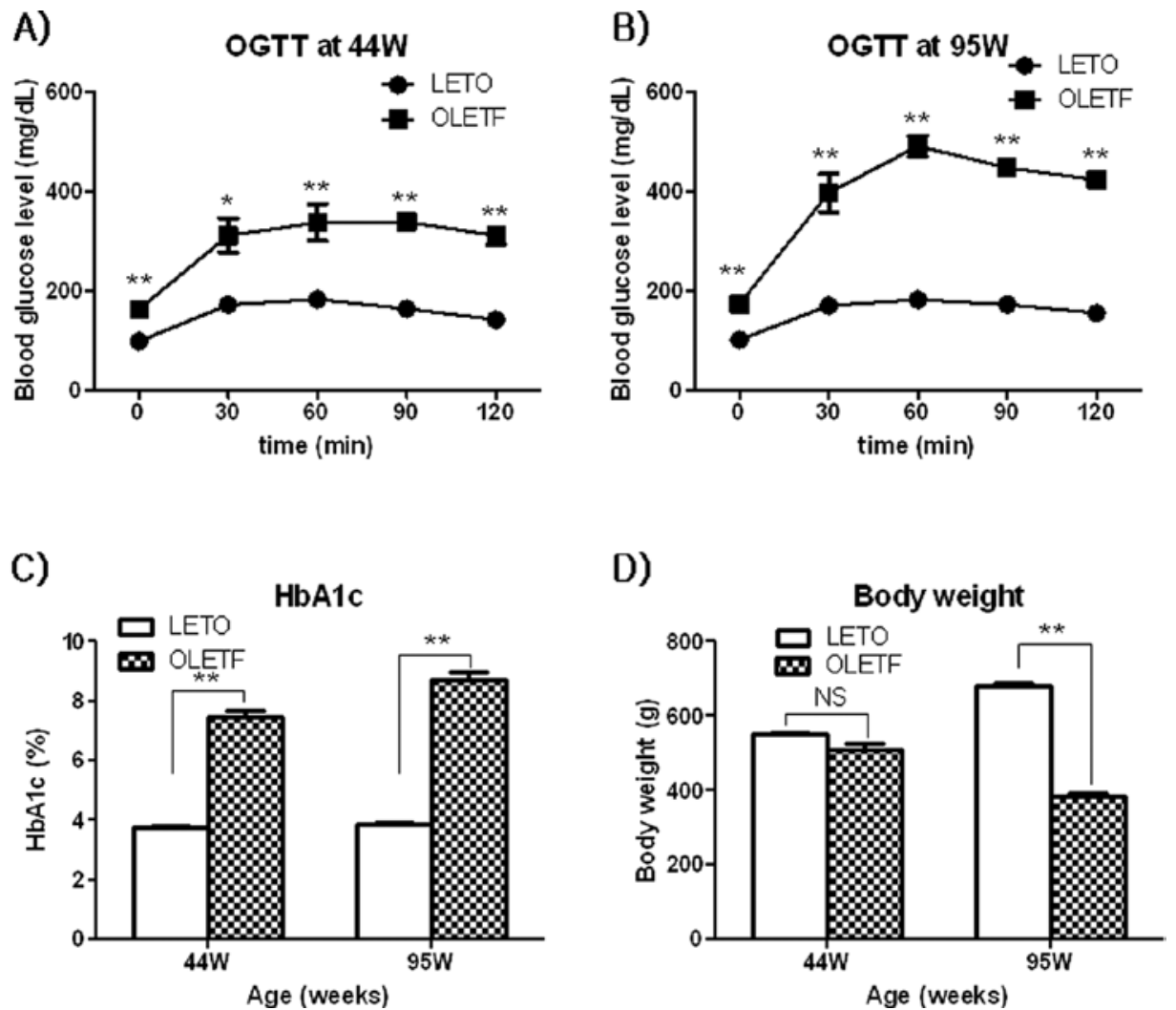

Figure 1. Indicators of diabetes in non-diabetic and diabetic rats. (Upper panels) Blood glucose levels as determined using oral glucose tolerance tests (OGTT) ( $2 \mathrm{~g}$ glucose/kg body weight) in the LETO and OLETF rats at (A) 44 and (B) 95 weeks of age. (C) HbA1c levels in control LETO rats and diabetic OLETF rats. (D) Body weights of LETO and OLETF rats at 44 and 95 weeks of age. ${ }^{*} \mathrm{P}<0.05,{ }^{* * *} \mathrm{P}<0.01$. NS, not significant.

used (seven 44-week-old rats and seven 95-week-old rats). The experimental animal protocol used in the study was approved by the Institutional Review Board of Kyung Hee University Hospital at Gangdong. All protocols concerning animal maintenance and handling were in accordance with NIH standards established in the Guidelines for the Care and Use of Experimental Animals.

Laboratory analyses of rat blood. Rats underwent an oral glucose tolerance test (OGTT) after an overnight fast. Rats were administered a glucose solution $(2.0 \mathrm{~g} / \mathrm{kg}$, p.o.), and blood samples were collected at 30, 60, 90 and $120 \mathrm{~min}$. Plasma glucose levels were determined by blood glucose sensors (MediSense Optium Xceed) and electrodes (Optium, Abbott Diabetes Care Inc., Alameda, CA, USA). Glycated hemoglobin (HbA1c) levels were measured using Siemens DCA Vantage ${ }^{\mathrm{TM}}$ analyzer (Tarrytown, NY). Rat body weight was also measured to compare diabetic with non-diabetic rats.

Histopathology. The knee and ankle joint including the entire foot were removed from each animal for histological analysis. From each rat, 2 knee joints and 4 foot joints were obtained. A total of 14 knee joints and 28 foot joints from rats of each group were histologically examined. Joints were dissected and fixed with $10 \%$ buffered formalin, and the paraffin-embedded sections were stained with hematoxylin and eosin (H\&E). Periodic acid-Schiff, methenamine silver, and Massontrichrome staining were also performed to clearly visualize blood vessel and collagen, respectively. All slides were viewed under a light microscope. The severity of the lesions was classified into 4 grades.

Statistical analysis. The experimental data are expressed as the mean \pm SEM. Differences between groups were compared using the Mann-Whitney test. Differences were considered significant at $\mathrm{P}<0.05$.

\section{Results}

To verify that OLETF rats had diabetes, we performed OGTT and measured HbA1c levels in OLETF and LETO rats. As shown in Fig. 1, the fasting blood glucose in the OLETF rat group was higher than that of the LETO rat group and the blood glucose level of OLETF rats was greatly elevated at 30, 60 and 90 min after glucose loading compared to that of LETO rats in both age groups (44 and 95 weeks). In addition, HbAlc was elevated in the OLETF as compared to the LETO rats in both age groups. In 95-week-old rats, the body weight in the OLETF group was significantly decreased when compared to the age-matched animals in the LETO group, even though no differences were detected in the 44-week-old groups. These data indirectly suggest that in the 95-week-old OLETF rats diabetes had persisted for at least 50 weeks in the rats.

To determine whether changes had occurred in tissues surrounding the knee or interphalangeal joints, tissues at these sites were examined by histopathology. Neither variable arterial changes, which have been a critical finding in $\mathrm{DM}$, nor neural degenerative changes were observed in the 


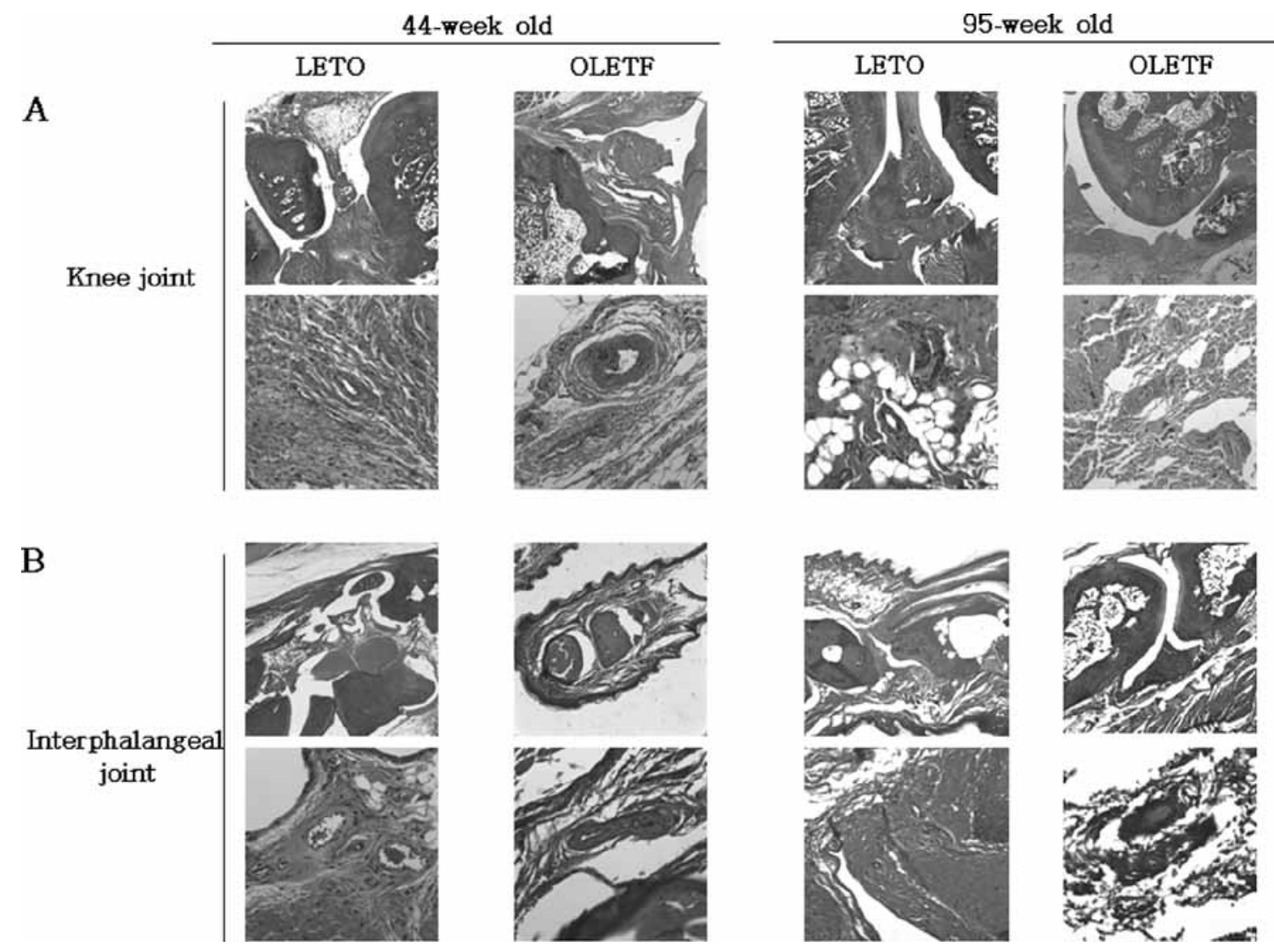

Figure 2. Comparison of arterial changes in tissues surrounding the (A) knee and (B) interphalangeal joints in 44- or 95-week-old rats (OLETF and LETO).

DM and the control animals in both the 44 and 95-week-old rat groups (Fig. 2). Furthermore, no evidence of necrosis of muscle fiber, phagocytosis of necrotic fibers, regeneration of muscle, mononuclear inflammatory cell infiltration, granulation tissue, calcification, and cartilage erosion was detected in any of the groups. Only mild degenerative changes with focal edema, collagen deposition and mild fibrosis were found in both the OLETF and LETO rats (95 weeks of age), particularly in the interphalangeal joints, although this outcome was not detected in the 44-week-old OLETF and LETO rats (data not shown).

\section{Discussion}

Hand stiffness is the most common event among various musculoskeletal manifestations of DM and accounts for approximately $30-76 \%$ of all musculoskeletal manifestations of DM $(7,8)$. It begins with flexion contracture in the joints of the little finger. However, no histological studies of the joints of patients with DM have been reported. Furthermore, the histopathological changes associated with hand stiffness have not been studied even in animal models of DM. The present study undertook an examination of these pathophysiological mechanisms and we expected to observe various arterial changes in and around the interphalangeal joints of rats with DM, which might give insights into the cause of stiff hands in humans with DM. However, we found no significant arterial changes in the OLETF rats.

Starkman et al reported arterial calcification in plain radiographs and a paucity of elastic fiber in the case of hand stiffness in diabetic patients (8). Another study demonstrated a clear link between hand and shoulder locomotor abnormalities in DM and evidence of poor glycemic control with an increased risk of other DM complications (9). We could not measure the stiffness of the phalangeal joints in OLETF rats but we did observe mild fibrosis in the interphalangeal joints, although it was not significantly different from that noted in the age-matched controls. Calcification or loss of elastic fiber could not be identified in this study. Focal degenerative change and fibrosis were noted in OLETF rats, but since the control group of LETO rats showed similar changes, these findings were attributed to age-related phenomena rather than a DM-associated manifestation. We found no other musculoskeletal manifestations. The lack of positive findings may reflect the low incidence of musculoskeletal manifestations in diabetes. Additionally, the life span of the rat may be too short to permit these histological alterations at the cellular level such as basal membrane thickening or advanced glycation endproduct (AGE) deposition in or around the joint. Thus, other diabetic rodent models need to be further studied for this purpose (10).

In conclusion, hand and shoulder disorders have been observed in patients with DM. Thus, the identification of characteristic musculoskeletal findings may facilitate earlier diagnosis of DM and initiation of treatment. This is the first report, to our knowledge, to examine musculoskeletal manifestations in an animal model of DM. However, to further understand the precise pathologic mechanisms that lead to hand and shoulder disorders in DM patients, additional investigations are required. 


\section{References}

1. Arkkila PE and Gautier JF: Musculoskeletal disorders in diabetes mellitus: an update. Best Pract Res Clin Rheumatol 17: 945-970, 2003.

2. Crispin JC and Alcocer-Varela J: Rheumatologic manifestations of diabetes mellitus. Am J Med 114: 753-757, 2003.

3. Gamstedt A, Holm-Glad J, Ohlson CG and Sundstrom M: Hand abnormalities are strongly associated with the duration of diabetes mellitus. J Intern Med 234: 189-193, 1993.

4. Lebiedz-Odrobina D and Kay J: Rheumatic manifestations of diabetes mellitus. Rheum Dis Clin North Am 36: 681-699, 2010.

5. Kawano K, Hirashima T, Mori S, Saitoh Y, Kurosumi M and Natori T: Spontaneous long-term hyperglycemic rat with diabetic complications. Otsuka Long-Evans Tokushima Fatty (OLETF) strain. Diabetes 41: 1422-1428, 1992.

6. Chen D and Wang MW: Development and application of rodent models for type 2 diabetes. Diabetes Obes Metab 7: 307-317, 2005.

7. Pal B, Anderson J, Dick WC and Griffiths ID: Limitation of joint mobility and shoulder capsulitis in insulin- and non-insulin-dependent diabetes mellitus. Br J Rheumatol 25: 147-151, 1986.

8. Starkman HS, Gleason RE, Rand LI, Miller DE and Soeldner JS: Limited joint mobility (LJM) of the hand in patients with diabetes mellitus: relation to chronic complications. Ann Rheum Dis 45: 130-135, 1986.

9. Ramchurn N, Mashamba C, Leitch E, et al: Upper limb musculoskeletal abnormalities and poor metabolic control in diabetes. Eur J Intern Med 20: 718-721, 2009.

10. Wu KK and Huan Y: Diabetic atherosclerosis mouse models. Atherosclerosis 191: 241-249, 2007. 